\title{
AGREEMENT BETWEEN PHYSICIAN RATING AND ON-ROAD DECISION FOR DRIVERS WITH MULTIPLE SCLEROSIS
}

\author{
Hannes Devos ${ }^{1}$, Maud Ranchet ${ }^{1}$, Mark Tant $^{2}$ \& Abiodun E. Akinwuntan ${ }^{1}$ \\ ${ }^{1}$ Georgia Regents University, Augusta, GA, USA \\ ${ }^{2}$ Belgian Road Safety Institute, Brussels, Belgium \\ hdevos@gru.edu
}

\begin{abstract}
Summary: The recommendation of the referring physician is paramount in the decision making process of fitness to drive for individuals with multiple sclerosis (MS). This medical advice is carefully considered by fitness to drive officials when making a final decision. In this study, we sought to determine the reliability between physician recommendation and decision of the on-road assessor in 95 individuals with MS. The percentage agreement $\left(\mathrm{p}_{\mathrm{o}}\right)$ and prevalence and bias adjusted kappa (PABAK) were used as measures of reliability. The on-road assessor found no concerns on the road in $87(92 \%)$ of the individuals; $6(6 \%)$ exhibited difficulties on the road that were of concern; and $2(2 \%)$ were advised to discontinue driving based on the findings of the road test. The $\mathrm{p}_{\mathrm{o}}$ between referring physician and on-road assessor was $83 \%$. The PABAK showed a reliability coefficient of 0.76 ( $\mathrm{p}<0.0001)$. No differences were found in $\mathrm{p}_{\mathrm{o}}$ between neurologists $(83 \%)$ and general practitioners $(88 \%$, Fisher's Exact $=0.56)$. Binocular acuity correlated significantly with the on-road driving decision (Spearman $\rho=-0.30 ; p=0.004)$. We conclude that, in this sample of drivers with MS, physicians were most of the time accurate in their appraisal of their patients' driving capabilities.
\end{abstract}

\section{INTRODUCTION}

Most developed countries have, to some extent, developed fitness to drive criteria for individuals with neurological conditions (Devos et al, 2012a). In many jurisdiction, a road test seems to constitute the core component of fitness to drive decisions (Devos et al, 2012a). In Belgium, drivers who report any significant change in medical status giving rise to nonconformity with the medical criteria stipulated in the law, must surrender their driver's license to the local authorities within four days of notice. In order to resume driving, a candidate driver with a neurological condition will need to obtain medical clearance for driving resumption by a neurologist. When in doubt or when there are functional deficits that require car adaptations, physicians refer the candidate driver to the Center for Evaluation of Fitness to Drive and Car Adaptations (CARA) of the Belgian Road Safety Institute. The referring physician will document the medical history and send along his recommendation with regard to fitness to drive. Therefore, physician documentation of the medical conditions and its potential impact on on-road driving are important inputs to the fitness to drive procedural system. Other countries have adopted a similar referral process (Devos et al, 2012a).

In most cases, the physician will make a recommendation based on the findings of a clinical examination and a (driving) interview with the patient and next of kin to appraise the patient's fitness to drive (Devos et al, 2012b). However, such evaluations may be inaccurate because 
patients fail to identify driving skills that are found to be problematic on a standardized on-road test (Wild \& Cotrell, 2003). Previous findings in other neurodegenerative conditions have shown that physicians tend to overestimate the fitness to drive of their patients and refer those with the poorest driving skills to the official authorities while the ones with more subtle impairments go unrecognized (Devos et al, 2012b; Heikkila et al, 1998).

It is unclear to what extent the referring physician recommendations are followed by fitness to drive officials in individuals with multiple sclerosis (MS). This condition is of particular interest due to the unpredictable progression and the heterogeneity in motor, visual, and cognitive symptoms (Compston \& Coles, 2008). Individuals with spasticity in the legs exhibit difficulties with pedal movements and maintaining lane positioning (Marcotte et al, 2008). Decreased color perception may impair the ability to distinguish road signs and traffic lights (Abiodun Emmanuel Akinwuntan et al, 2013). Drivers with cognitive impairments are more likely to be involved in car crashes and incur a significantly higher number of traffic offences than do control drivers and drivers with MS who have no apparent cognitive impairments (Schultheis et al, 2002). Accurate referral from first-line physicians to the fitness to drive evaluation center is therefore paramount to discern drivers with MS who are safe on the road from those who may potentially endanger themselves and other road users.

In this cross-sectional study, we sought to better understand the observations and concerns from referring physicians with regard to the fitness to drive of their patients with MS. Three questions were addressed: (1) What is the agreement in fitness to drive decision between the referring physician and the result of the on-road driving assessment? (2) Are there differences in the levels of agreement between general practitioners and neurologists? (3) To what extent do demographic, clinical, and driving characteristics play a role in the referring physician's decision and in the on-road decision?

\section{METHOD}

\section{Participants}

A random sample of clients diagnosed with MS who underwent an official fitness to drive evaluation from July 2012 to December 2013 at the CARA Department of the Belgian Road Safety Institute were included in this study. As this was a population-based study, no specific inclusion and exclusion criteria were identified a priori. The conduct of the study was approved by the Institutional Review Board (IRB-A) of Georgia Regents University.

\section{Procedure}

Referring physician. The medical and driving history questionnaire was mailed to the clients who requested a fitness to drive evaluation. The questionnaire was filled out by the candidate and the referring physician, signed, and sent back to CARA prior to scheduling an appointment. The referring physician was chosen by the candidate driver and could be a general practitioner or a neurologist. The medical and driving questionnaire comprised detailed information on the type of medical condition, medication use, and the number of traffic violations and car crashes in the last five years. The questionnaire also contained information on the type of application: if the 
drivers reported (1) a change in medical status; (2) an extension of the validity date of their driver's license; (3) a medical clearance to obtain a new driver's license; or (4) if there was a mandatory referral by the insurance company, court, or medical expert. The referring physician was asked to give an advice regarding the fitness to drive into three categories: favorable; reserved; or unfavorable. Candidates were assigned to the favorable category if they were deemed fit to drive without or with adaptations (e.g., automatic transmission, left sided accelerator pedal, controls on steering wheel), but with no restricted use in time (e.g., daylight only), distance (e.g. familiar area), or speed (e.g., no highways). Candidates assigned to the reserved category were also allowed to continue driving with or without adaptations, but with restricted use in time, distance, or speed. Finally, the unfavorable category comprised the individuals who were advised to stop driving. The referring physician was blind to the outcome of the road test and vice versa.

On-road drive. The 20-km standardized road test was performed between 9 AM and 4 PM with a car from CARA fitted with dual controls to ensure driving safety. Vehicle adaptations were used if needed. For example, the pedals were replaced by hand controls in case of lower extremity weakness. The right sided accelerator pedal was replaced by a left sided accelerator pedal in case of weakness or spasticity in the right leg. In Belgium, it is not the habit to drive a car with automatic transmission. Therefore, if there were no motor or cognitive contra-indications, a car with manual transmission was used. Clients who were evaluated in a car with automatic transmission were no longer allowed to continue driving a car with manual transmission. Clients were first instructed to adjust the driver's seat and mirrors. To familiarize themselves with the steering wheel and pedals, clients drove two laps on a closed course. The actual on-road test started in a suburban area with low to moderate traffic, progressed to a 6-lane, 2-way highway, continued to an urban area, and terminated at the driving evaluation center. The on-road instructor was an occupational or physical therapist certified to conduct fitness to drive evaluations. The on-road instructor was seated in the passenger seat and operated the safety controls, gave directions to the candidates, and evaluated the impact of distraction on on-road driving by engaging the client in conversations at regular time intervals. After the road test, participants were classified into the same three categories as described above. Of note, in Belgium, the final fitness to drive decision is based on medical, clinical, visual, on-road, and in some cases, neuropsychological tests. For easy comparison with countries that use a road test as single criterion of fitness to drive and because road tests explain most of the variance of the final fitness to drive decision (Akinwuntan et al, 2002), we used the findings of the road test as the main outcome variable.

Visual tests. Binocular acuity was administered using the Ergovision equipment to determine whether the candidate driver met the legal visual requirements of 20/40 of binocular vision. The scale ranged from 0 to 12 .

\section{Data analysis}

Percentage of agreement $\left(\mathrm{p}_{\mathrm{o}}\right)$ and Prevalence and Bias Adjusted Kappa (PABAK) for ordinal scales (Vannest, Parker, \& Gonen, 2011) were calculated to measure the interrater reliability between the recommendation of the referring physician and the on-road outcome. The PABAK for ordinal values is defined as the chance-adjusted percent of agreement on an ordinal scale, 
including partial credit for near misses. The interpretation of the PABAK is similar to the weighted kappa statistic (Byrt, Bishop, \& Carlin, 1993). PABAK values of $0-0.20$ indicated slight agreement, $0.21-0.40$ fair, $0.41-0.60$ moderate, $0.61-0.80$ substantial, and $0.81-1$ almost perfect agreement (Landis \& Koch, 1977). Differences in the observed percentage of agreement $\left(\mathrm{p}_{\mathrm{o}}\right)$ between neurologists and general physicians were calculated using Chi-square tests. Medical, clinical, and driving variables were correlated with the refferal rating and the onroad decision using Spearman $\rho$ statistics and rank biserial statistics. P values $<0.05$ were considered significant. All statistical procedures were performed using SAS, version 9.1, Cary, NC.

\section{RESULTS}

Demographic, driving, and disease characteristics of the 95 individuals with MS are displayed in Table 1. Twenty-eight (29\%) reported comorbidity including visual deficits $(n=12)$; depression $(\mathrm{n}=3)$; history of epilepsy $(\mathrm{n}=2)$; sleep apnea $(\mathrm{n}=2)$; drug abuse $(\mathrm{n}=2)$; diabetes mellitus type $2(n=2)$; hearing loss $(n=2)$; liver and kidney disease $(n=2)$; and discus hernia $(n=1)$.

Twenty-six $(27 \%)$ were first-time visitors to CARA, the remainder $(n=69,73 \%)$ had visited CARA prior to this assessment.

Table 1. Demographic, driving, and clinical characteristics of the MS group $(n=95)$

\begin{tabular}{lcc}
\hline Variable & Central tendency measure & Range \\
\hline Age, y & $53 \pm 12$ & $26-82$ \\
Sex, male (\%) & $44(45)$ & N/A \\
Type of application* & $25(27) / 62(67) / 2(2) / 4(4)^{* *}$ & N/A \\
Number of car crashes in past 5 years, $\mathrm{n}$ & $0(0-0)$ & $0-2$ \\
Number of traffic violations in past 5 years, & $0(0-0)$ & $0-6$ \\
Driving experience, y & $3(2-10)$ & $0-44$ \\
Disease duration, y & $7(6-18)$ & $0-35$ \\
Binocular acuity, /12 & $10(8-10)$ & $5-12$
\end{tabular}

Results are presented as means \pm standard deviation, medians (Q1 - Q3) or frequencies (\%).

Abbreviation: MS, Multiple Sclerosis.

* Change in medical condition / extension of license / new license / mandatory referral

** Missing data: $\mathrm{n}=2$.

The on-road assessor allocated $87(92 \%)$ to the favorable category, $6(6 \%)$ to the reserved category, and $2(2 \%)$ to the unfavorable category. No differences in favorable decisions were found between first-time visitors $(24 / 26,92 \%)$ and those who had visited CARA before (63/69, 91\%; Fisher's Exact, $\mathrm{p}=0.80$ ). Of the 93 clients who could resume driving based on the outcome of the road test, $51(55 \%)$ were advised to drive a car with automatic transmission. The accelerator and brake pedals were replaced with hand controls in $20(22 \%)$ of those who could continue driving with automatic transmission. Three (3\%) persons who required automatic transmission needed a spinner knob. 
The referring physician allocated $85(89 \%)$ in the favorable category, $8(8 \%)$ in the reserved category, and $2(2 \%)$ in the unfavorable category.

The comparison of the physician rating with the on-road decision is found in Table 2 . The physician and on-road assessor agreed on $80\left(p_{0}=83 \%\right)$ of the cases. Of the 15 persons that the physician and on-road assessor did not agree upon, six were considered favorable by the referring physician whereas the on-road driving assessor advised restrictions in four individuals and even driving cessation in two. Conversely, the physician was more stringent than the on-road driving assessor in nine cases.

When adjusted for prevalence and bias, the PABAK between physician rating and the on-road decision was $0.76(0.67-0.86 ; \mathrm{p}<0.0001)$.

Table 2. Contingency table of physician recommendation with on-road decision

\begin{tabular}{lllll}
\hline & \multicolumn{3}{c}{ On-road decision } & Totals \\
\cline { 2 - 4 } Physician recommendation & Favorable & Reserved & Unfavorable & 85 \\
Favorable & 79 & 4 & 2 & 8 \\
Reserved & 7 & 1 & 0 & 2 \\
Unfavorable & 1 & 1 & 0 & 95 \\
Totals & 87 & 6 & 2 & \\
\hline
\end{tabular}

The type of referring physician (general practitioner versus neurologist) was unknown for 6 cases. The percentage of accurate classifications was similar for neurologists $(34 / 41 ; 83 \%)$ and general practitioners $(42 / 48 ; 88 \%$, Fisher's Exact $=0.56)$. No significant correlations were found between the physician recommendation and any of the variables listed in Table 1 . The on-road decision correlated significantly with binocular acuity $(\rho=-0.30 ; p=0.004)$. No other demographic, driving or clinical variables correlated with the on-road decision.

\section{DISCUSSION}

The majority of drivers with MS in this study were found to be safe on the road. Only two patients with MS who were actively driving at the moment of testing were advised to stop driving after the road test. Six more were advised to continue driving but with restrictions of use in time, distance or speed. Although the majority were considered to be fit to drive with no restrictions based on the findings of the road test, a substantial proportion needed car modifications to compensate for their loss of motor functions.

The percentage of agreement and the PABAK showed substantial agreement between the referring physician and the results of the road test. This finding indicates that either the appraisal of the physician is closely followed by the on-road assessor or that the referring physician has the proper skills to determine the driving competences of their patients. More than $80 \%$ of the patients were correctly classified by the referring physician. This percentage accuracy is slightly lower than any clinical battery developed so far to screen for fitness to drive in MS (Akinwuntan et al, 2013; Lincoln \& Radford, 2008). However, the physician ratings were less reliable for drivers who exhibited difficulties on the road: four out of six with concerns on the road and two 
who were advised to stop driving were not correctly categorized by the referring physician. Future studies should investigate whether the appraisal of the physician in combination with the outcome of a clinical screening battery can further reduce the number of incorrectly classified individuals.

The present findings contradict previous work in other neurodegenerative conditions that showed at best moderate agreement between physicians and fitness to drive decisions (Devos et al, 2012b; Heikkila et al, 1998; Ott et al, 2005). The reason for this discrepancy may be that individuals with MS are followed up more closely with regard to their fitness to drive. They are generally younger and more likely to be using their car for work and leisure (Neven et al, 2013). Consequently, individuals with MS may present with a better cognitive and driving profile than drivers with Alzheimer disease (AD), Parkinson disease (PD), or Huntington disease (HD). Also, in contrast to neurodegenerative conditions, the progression of MS is in the majority of individuals relapsing-remitting, i.e., exacerbations of symptoms followed by gradual amelioration of symptoms (Compston \& Coles, 2008). Physicians may be more vigilant in evaluating fitness to drive after a relapse than when there is a slow deterioration of motor and cognitive functions as observed in AD or PD. Finally, one in five $(20 \%)$ of drivers with MS needed substantial car modifications (hand controls, spinner knob) due to the nature of their motor problems (e.g., spasticity, muscle weakness). It may that in this population-based sample of MS, the majority were referred because of motor problems that could be compensated with car adaptations and did therefore not interfere with their fitness to drive. Clients with AD, PD, or HD usually cannot benefit from car adaptations other than automatic transmission to compensate for their motor symptoms. Future studies should focus on the comparison of on-road scores, cognitive performance, and reasons for referral between these neurological conditions.

Neurologists were not more accurate in predicting the outcome of the road test than general practioners. Previous work in AD suggested that physician-specialists were better suited to evaluate fitness to drive than health care professionals with no specialized training (Ott et al, 2005). This finding was not confirmed in our study.

None of the demographic, medical, clinical, and driving data recorded in the questionnaire correlated with the physician recommendation. It therefore remains unclear what factors constitute the physician recommendation. In contrast, binocular acuity correlated significantly, yet only moderately, with the on-road decision. This last finding is in accordance with several studies that showed cognitive functions to be more predicitive of performance in the road test than motor or basic visual sensory measures (Akinwuntan et al, 2013; Lincoln \& Radford, 2008).

The main limitation is that all subjects had their own and therefore different referring physician. We can not determine what the effect is of experience, knowledge of fitness to drive regulations, or relationship with the patient, on their recommendation of fitness to drive. Further research on the association between first-tier referrals and on-road decisions is therefore warranted.

\section{ACKNOWLEDGMENTS}

The authors thank the CARA staff for the fitness to drive evaluations. We also express our sincerest thanks to Erin Neal, BA for technical and administrative assistance. 


\section{REFERENCES}

Akinwuntan, A. E., Devos, H., Stepleman, L., Casillas, R., Rahn, R., Smith, S., \& Williams, M. J. (2013). Predictors of driving in individuals with relapsing-remitting multiple sclerosis. Multiple Sclerosis Journal, 19(3), 344-350.

Akinwuntan, A. E., Feys, H., De Weerdt, W., Pauwels, J., Baten, G., \& Strypstein, E. (2002). Determinants of driving after stroke. Archives of Physical Medicine and Rehabilitation, 83(3), 334-341.

Byrt, T., Bishop, J., \& Carlin, J. B. (1993). Bias, prevalence and kappa. Journal of Clinical Epidemiology, 46(5), 423-429.

Compston, A., \& Coles, A. (2008). Multiple sclerosis. Lancet, 372(9648), 1502-1517.

Devos, H., Akinwuntan, A. E., Gelinas, I., George, S., Nieuwboer, A., \& Verheyden, G. (2012a). Shifting up a gear: considerations on assessment and rehabilitation of driving in people with neurological conditions. Physiotherapy Research International, 17(3), 125-131.

Devos, H., Nieuwboer, A., Tant, M., De Weerdt, W., \& Vandenberghe, W. (2012b). Determinants of fitness to drive in Huntington disease. Neurology, 79(19), 1975-1982.

Heikkila, V. M., Turkka, J., Korpelainen, J., Kallanranta, T., \& Summala, H. (1998). Decreased driving ability in people with Parkinson's disease. Journal of Neurolology Neurosurgery and Psychiatry, 64(3), 325-330.

Landis, J. R., \& Koch, G. G. (1977). The Measurement of observer agreement for categorical data. Biometrics, 33(1), 159-174.

Lincoln, N. B., \& Radford, K. A. (2008). Cognitive abilities as predictors of safety to drive in people with multiple sclerosis. Multiple Sclerosis, 14(1), 123-128.

Marcotte, T. D., Rosenthal, T. J., Roberts, E., Lampinen, S., Scott, J. C., Allen, R. W., \& CoreyBloom, J. (2008). The contribution of cognition and spasticity to driving performance in multiple sclerosis. Arch Phys Med Rehabil, 89(9), 1753-1758.

Neven, A., Janssens, D., Alders, G., Wets, G., Wijmeersch, B. V., \& Feys, P. (2013). Documenting outdoor activity and travel behaviour in persons with neurological conditions using travel diaries and GPS tracking technology: a pilot study in multiple sclerosis. Disability and Rehabilitation, 35(20), 1718-1725.

Ott, B. R., Anthony, D., Papandonatos, G. D., D'Abreu, A., Burock, J., Curtin, A., Wu, C. K., Morris, J. C. (2005). Clinician assessment of the driving competence of patients with dementia. Journal of the American Geriatrics Society, 53(5), 829-833.

Schultheis, M. T., Garay, E., Millis, S. R., \& Deluca, J. (2002). Motor vehicle crashes and violations among drivers with multiple sclerosis. Archives of Physical Medicine and Rehabilitation, 83(8), 1175-1178.

Vannest, K. J., Parker, R. I., \& Gonen, O. (2011). Single Case Research: web based calculators for SCR analysis. (Version 1.0) [Web-based application]. Available from singlecaseresearch.org, retrieved October 29, 2014.

Wild, K., \& Cotrell, V. (2003). Identifying driving impairment in Alzheimer disease: a comparison of self and observer reports versus driving evaluation. Alzheimer Disease and Associated Disorders, 17(1), 27-34. 